\title{
Capacity and Fairness of the Multiple Access Channel in Energy Harvesting Wireless Networks
}

\author{
R.A. Raghuvir, Dinesh Rajan, Senior Member, IEEE, Mandyam D. Srinath, Senior Member, IEEE \\ Department of Electrical Engineering, Southern Methodist University, Dallas, TX 75275
}

\begin{abstract}
In this paper, we obtain the capacity region for the AWGN Multiple Access Channel (MAC) with transmitters equipped with energy harvesters. We then develop low delay, adaptive transmission strategies that surprisingly achieve near optimal performance under finite battery resource constrained settings for the flat fading MAC. We describe the rate-fairness tradeoff inherent to the MAC and adapt the well known proportionally fair (PF) rate allocation to energy harvesting nodes. We develop new adaptive transmission strategies based on the $P F$ rate allocation that achieves points above the rate-fairness boundary of the convex hull of the PF rate allocation.
\end{abstract}

Index Terms-Multiple Access Channel, Energy harvesting, Jain fairness index, Proportionally Fair Rate Allocation, MaxSNR

\section{INTRODUCTION}

The ergodic capacity of the additive white Gaussian noise (AWGN) channel with an average transmit power constraint, is first given by Shannon in his pioneering work [1]. Subsequently, the capacity of several multiuser fading channels have been widely investigated (see [2] for an excellent review). Specifically, the capacity region of a fading multiple access channel (MAC) is elegantly characterized in [3] and shown to have a polymatroidal shape. In the special case of achieving the maximum sum-rate point, an adaptive TDMA strategy, that picks a single user with the best channel for every channel use, is shown to be optimal [4]. This scheme is commonly known as the MaxSNR scheme.

One of the well known drawbacks associated with the optimal sum-rate maximizing strategy, is poor fairness of the rate allocation. Users in poor channel conditions, but with delay sensitive data, may not get serviced in a timely manner. Researchers have investigated several algorithms such as the proportional fairness (PF) scheme [5], improve the fairness of the rate allocation, in multi-user scenarios. Trade-offs between rate and fairness for the MAC can be achieved by varying a window size parameter in the PF scheme.

Recently, several information-theoretic analyses of channels with an energy harvesting mechanism at the transmitter have appeared in the literature. The capacity of a point-to-point AWGN channel with an energy harvester at the transmitter is given in [6], [7], [8]. In contrast to traditional capacity results which are typically derived under an average transmit power constraint, in the energy harvesting scenario, the energy harvesting process induces a constraint on the transmit power at each time epoch. In these works, the capacity is shown to be upper bounded by the capacity of a point-to-point system without an energy harvester at the transmitter, but with average power constraint equal to the recharge rate.

In this paper, we investigate the performance of a MAC when the individual transmitters are equipped with energy harvesters and a finite energy storage element such as a battery. We study the Shannon theoretic capacity of the AWGN MAC with an energy harvester at each transmitter. For the flat fading MAC, we consider low delay schemes that attempt to achieve the maximum sum-rate point, and compare them to the optimal MaxSNR scheme. In a MAC, where the individual transmitters are equipped with energy harvesters and a finite energy storage element such as a battery, jointly using the knowledge of channel state, battery conditions and instantaneous fairness values become important in the design of adaptive transmission strategies.

The main contributions of this paper are as follows. First, we characterize the capacity of a AWGN-MAC with an energy harvester at each transmitter and an infinite capacity battery. We show that this capacity region is the same as the capacity region of a non-energy-harvesting MAC with equivalent average power constraints for each user. Second, for the fading MAC we propose three adaptive, low delay transmission strategies that achieve rates close to the maximum sum-rate point under certain scenarios. We show that when the individual users have finite size batteries to store their harvested energy, the scheme that achieves a rate close to the maximum sum-rate point, is one that allows a group of users to transmit simultaneously for every channel use. Transmission by a single user with the best channel is no longer optimal in the low delay, resource constrained settings, with energy harvesting transmitters. Third, we adapt the PF rate allocation scheme to energy harvesting networks (and term it PFEH) and propose a new adaptive transmission strategy that achieves better throughput and fairness over the PFEH scheme, i.e., the rate-fairness points achieved are above the convex hull of the boundary achieved by PFEH. Significantly, these new results hold for non-energy harvesting MAC as well. Fourth, we characterize, through extensive simulation, the throughput-fairness performance of all the low delay, adaptive transmission strategies under various conditions such as (a) low and high SNR (or recharge rate) (b) small and large battery sizes (c) few and many users and (d) identical (symmetric) and different (asymmetric) recharge rates for the users. We show that, in the small battery regime, schemes that consider the battery state in addition to the channel state when a single user transmits, and schemes that consider a group of users, achieve higher sum-rate than schemes that consider the channel alone. 
Our new scheme that outperforms the PFEH scheme in terms of the rate-fairness boundary, achieves better fairness with only a small penalty in lost rate, when compared to schemes that maximize the sum-rate point.

The results in this paper are applicable in a wide range of scenarios, for instance in a wireless sensor network, where each node has an energy harvester and is communicating with a fusion center. We consider centralized scheduling and power control at the fusion center, in which the fusion center instructs each sensor node as to when to transmit and the transmit power level to be used. We also assume that a feedback channel provides accurate information of the instantaneous channels and battery conditions of the various users to the fusion center. A variety of metrics have been used in the literature to measure fairness of resource allocation towards the users. We adopt the Jain Index of Fairness in our work [9]. Other metrics for fairness that are applicable to the physical layer are described in [10]. Note that any of these metrics can be directly incorporated into our simulation studies.

\section{A. Related Work}

In a flat fading channel, the channel gain or channel side information (CSI), stays constant during a symbol interval. The capacity of such a channel with CSI known to both transmitter and receiver, and with average power constraint on the transmitter, is given in [11]. The power allocation strategy that achieves capacity is the well known water-filling in time, which takes advantage of good channel conditions.

In a multi-user flat fading multiple access channel, the optimal power control that maximizes the sum-of-rates is shown to be a TDMA-like approach [4] popularly known as opportunistic scheduling [12]. In this approach, only the user that enjoys the best channel gain is allowed to transmit and the assigned power is computed by temporal water-filling, similar to the single user scenario [11]. In [13], opportunistic scheduling is considered in energy harvesting networks with emphasis on queue stability rather than sum-of-rates capacity. The variation of mean delay with the packet arrival rate is characterized for certain decentralized algorithms.

The well known proportionally fair scheme is proposed in [5] for the down-link channels and subsequently adapted for the up-link in the context of a CDMA packet data system in [14]. We use a more structured framework for the rate allocation using the marginal utility function developed in [3] than [14].

Trade-off between rate and fairness have been considered in [15] for the down-link in cellular systems. In [15], a ratefairness trade-off is obtained by grouping the users based on different criteria and allowing a single user to access the channel. In contrast, we obtain a rate-fairness trade-off for the up-link channel, by allowing more than one user to compete for channel access using a preferential weighting to selected users based on normalized rate. Moreover, these works do not consider energy harvesting nodes, which is the focus of this work.

The capacity of the single user AWGN channel with an energy harvester at the transmitter, is given in [6], [7], [8]. Our work differs from these works by the fact that we consider the multi-user scenario of a multiple-access channel. We extend the results in [6], [7] to the multi-user case, and study ratefairness trade-offs which are not studied in those works. The work in [8] was extended in [16] to find the capacity of a fading channel in energy harvesting systems. The optimal power allocation strategy in this case is also shown to be water-filling in time, with the average power constraint equal to the average recharge rate. The two user MAC for energy harvesting systems has been investigated recently in [17]. However the authors consider optimal off-line policies where the energy arrival times and the amount of energy harvested are known apriori. A variety of multi-user scenarios have been considered in recent work for relay [18], broadcast [19] and interference [20] channels, wherein the authors discuss the optimality of off-line scheduling policies and sub-optimal online policies. Our work considers on-line policies where only the statistics of the energy harvesting process (such as average recharge rate) is known apriori. On-line policies for single user energy harvesting systems have been proposed in [21], however multi-user scenarios are not considered. In contrast to these works, our work is the first to jointly consider rate and fairness in multi-user energy harvesting wireless networks.

The rest of the paper is organized as follows. Section III gives the capacity region for the AWGN-MAC with energy harvesting transmitters. In Section III] we describe the characterization of the capacity region of the flat fading Multiple Access Channel and adapt the underlying framework to energy harvesting systems. In Section IV] we discuss the index of fairness and the proportionally fair rate allocation. In Section $\mathrm{V}$ we describe several low delay, adaptive transmission strategies. Numerical results are presented in Section VI. Section VII concludes the paper.

\section{CAPACity REgion OF THE AWGN MAC}

Consider a discrete time multiple access channel with $K$ users transmitting to a single receiver. Let $X_{i, j}$ represent the signal transmitted by user $i$ at time instant $j$. The received signal $Y_{j}$ at the receiver is given by

$$
Y_{j}=\sum_{i=1}^{K} h_{i, j} X_{i, j}+N_{j},
$$

where $h_{i, j}$ is the channel gain of user $i$ at time $j$ and $N_{j}$ is the additive noise at the receiver which is modeled as Gaussian with zero mean and variance $\sigma^{2}$. For a non-faded MAC, $h_{i, j}=$ $h_{i} \forall j$.

Each user $i$ has an energy harvesting mechanism and the energy harvested at time $j$ is denoted by $E_{i, j}$ Joules. The energy harvesting process for each user is assumed to be ergodic and independent of the energy harvesting of the other users. Let the symbol period equal $T$. Assume that each user also has a battery with capacity equal to $B$ Joules in which the harvested energy is stored. In the initial capacity results, we set $B \rightarrow \infty$. In subsequent analysis, we quantify the effect of finite battery sizes. This energy harvesting process induces a natural constraint on the transmit power up to any given time 
$m$ as,

$$
\sum_{j=1}^{m} X_{i, j}^{2} T \leq \sum_{j=1}^{m} E_{i, j} \quad \forall m, i=1,2, \ldots K
$$

For notational convenience, we define $C(x)=0.5 \log (1+x)$.

We first consider the case of a AWGN-MAC and characterize the capacity region in the following theorem.

Theorem 1: (Capacity Region of AWGN channel with Average Power Constraint) Consider the $K$-user AWGN MAC with independent random energy arrivals for each of the $K$ users represented by the vector $\left[E_{1} E_{2} \ldots E_{K}\right]$. Let $P_{i}=\mathbf{E}\left[E_{i}\right] / T$ represent the average power constraint for user $i$. Then, the transmission rates $R_{i}$ for user $i$ are given by the closure of the convex hull of the rate vectors satisfying

$$
\sum_{i \in S} R_{i} \leq C\left(\frac{\sum_{i \in S} h_{i}^{2} P_{i}}{\sigma^{2}+\sum_{i \in S^{c}} h_{i}^{2} P_{i}}\right) \forall S \subseteq\{1,2, \cdots, K\}
$$

where $S$ is the set of users and $S^{c}$ denotes the complement set.

Proof:

Upper bound: It is clear that the rates in (3) is the upper bound of a Gaussian MAC without energy harvesting and an average power constraint $P_{i}, \forall i$. Due to the i.i.d nature of $E_{i}$, and invoking the law of large numbers [7], $\frac{1}{n} \sum_{i=1}^{n} E_{i} \rightarrow P_{i}$. Hence, (3) is also an upper bound on the achievable rates with energy harvesting nodes.

Achievability:

For each user $i$ with energy harvesting, the maximum achievable rate is given in [6], [7], [8] as

$$
R_{i}=C\left(\frac{h_{i}^{2} P_{i}}{\sigma^{2}}\right)
$$

This rate is achieved using the save-and-transmit scheme employed in [6], [7]. Rather than ensuring that the cumulative power constraint in 2 is satisfied for every time instant, the random nature of the energy arrivals is smoothed out by storing the energy in an infinite size energy buffer. The transmitter essentially waits for $s(n)$ time slots before the start of transmission. It is shown in [6], [7] that $s(n) \in o(n)$ i.e. $s(n)$ belongs to a class of functions $o(n)$ that scale slower than $n$. This delayed transmission guarantees adequate energy is available in the energy buffer of each user and avoids the case of the encoder not being able to transmit a codeword due to insufficient energy. Further, in the limit of large $n$ the loss in rate due to non-transmission during the first $o(n)$ symbols reduces to 0 . We now extend this idea to the multiuser scenario.

Consider any set $S \subseteq\{1,2, \cdots, K\}$ of users. Depending on the arrival process for each user $i \in S$, using the results from [6], [7] there exists a time denoted by $s_{i}$ such that if user $i$ is silent for the first $s_{i}$ time instants, it can subsequently transmit at average power $P_{i}$ for the remaining $n-s_{i}$ time instants. Now, consider $\tilde{s}(S)=\left(\max \left(s_{i}\right), i \in S\right)$. Since each $s_{i} \in o(n)$, this implies that $\tilde{s}(S) \in o(n)$. Clearly, if all users $i \in S$ are silent for the first $\tilde{s}(S)$ time instants, they can subsequently transmit at their average powers. Now, let each user $i \in S$ transmit using an independent Gaussian code book (the traditional MAC capacity achieving code book without energy harvesting) of size $\left(2^{(n-\tilde{s}) R_{i}}, n-\tilde{s}\right)$. At the receiver, using the successive decoding method, any of the corner points of the capacity region can be achieved by carefully designating the order of decoding. Thus, any set of rates satisfying (3) can be achieved with arbitrarily small probability of error. Due to the transmitters remaining silent for duration $\tilde{s}$, the average rate achieved is lower and equals $\frac{n-\tilde{s}}{n} R_{i} \rightarrow R_{i}$ in the limit of large $n$.

\section{CAPACITY REgION OF THE FLAT FADING MAC}

For non-energy harvesting systems, [3] characterizes the capacity region $\mathbf{C}(\mathbf{P})$ for the fading $\mathrm{MAC}$, where $\mathbf{P} \in \mathcal{R}_{+}^{K}$ is the vector of average power constraints for the different users, and $\mathcal{R}_{+}^{K}$ is the set of all real positive numbers. The transmitters employ dynamic power control in response to the varying channel state in the optimal solution, when the channel state information is known to all the transmitters and the receiver.

Let $\mathbf{R}$ be a vector of rates. Then taking the average power constraint into consideration, the boundary surface of the capacity region can be found by casting the problem as a Lagrangian optmization problem as follows:

$$
\max _{(\mathbf{R}, \mathbf{P}) \in S_{r}} \mu \cdot \mathbf{R}-\lambda \cdot \mathbf{P}
$$

where

$$
S_{r}=\left\{(\mathbf{R}, \mathbf{P}): \mathbf{P} \in \mathcal{R}_{+}^{K}, \mathbf{R} \in \mathbf{C}(\mathbf{P})\right\}
$$

In (5), we can consider $\mu \in \mathcal{R}_{+}^{K}$ to be the vector of rate rewards that assigns priorities to the users, and $\lambda \in \mathcal{R}_{+}^{K}$, the vector of Lagrange multipliers, to be the set of power prices. For a given $\mu$, the value of $\lambda$ is chosen such that the average power constraint is satisfied. As $\mu$ varies, all points on the boundary of the capacity region are obtained.

We next briefly outline the iterative procedure used to compute the power prices $\lambda$, for a given vector of rate rewards $\mu$.

Expressing $\mathbf{R}$ explicitly in terms of $\mu$ in (5), the boundary of the capacity region of the Gaussian multiple access channel is shown in [3] (Theorem 3.16) to be the closure of the parametrically defined surface

$$
\left\{\mathbf{R}(\mu): \mu \in R_{+}^{K}, \sum_{i} \mu_{i}=1\right\}
$$

where $\forall i=1, \cdots, K$

$R_{i}(\mu)=\int_{0}^{\infty} \frac{1}{2\left(\sigma^{2}+z\right)}\left\{\int_{\frac{2 \lambda_{i}\left(\sigma^{2}+z\right)}{\mu_{i}}}^{\infty} f_{i}(h) \prod_{k \neq i} F_{k}\left([\alpha]^{*}\right) d h\right\} d z$

with

$$
\alpha=\frac{2 \lambda_{k} h\left(\sigma^{2}+z\right)}{2 \lambda_{i}\left(\sigma^{2}+z\right)+\left(\mu_{k}-\mu_{i}\right) h}
$$

and the []$^{*}$ operator is defined as in [22] as

$$
[x]^{*}= \begin{cases}x, & \text { if } x \geq 0 \\ +\infty & \text { if } x<0 .\end{cases}
$$


The vector $\lambda=\left\{\lambda_{i} \in R_{+}: i=1,2, \cdots, K\right\}$ is the solution of the equations

$$
\begin{aligned}
\int_{0}^{\infty}\left\{\int_{\frac{2 \lambda_{i}\left(\sigma^{2}+z\right)}{\mu_{i}}}^{\infty} f_{i}(h) \prod_{k \neq i} F_{k}\left([\alpha]^{*}\right) d h\right\} d z=P_{i} \\
\forall i=1,2, \cdots, K
\end{aligned}
$$

which represent the appropriate average power constraint for each user.

The procedure used to solve for $\lambda$ given $\mu$ can be expressed in terms of (10). If $\lambda(0)$ is the initial arbitrary set of power prices, and the $n$th iterate is represented by $\lambda(n)$, the $(n+1)$ th iterate $\lambda_{i}(n+1)$ of user $i$ is given by the unique root $x$ of the equation

$$
\int_{0}^{\infty}\left\{\int_{\frac{2 x\left(\sigma^{2}+z\right)}{\mu_{i}}}^{\infty} f_{i}(h) \prod_{k \neq i} F_{k}\left([\alpha]^{*}\right) d h\right\} d z=P_{i}
$$

and can be solved by binary search (see Algorithm 4.2.2 in [3]).

\section{A. The marginal utility function}

The rate achieved and the power used by the optimal solution in (5) can be calculated using the marginal utility function [3]. We show how the optimal solution in (5) can be expressed in terms of the marginal utility function. We then adapt the marginal utility function to energy harvesting systems. This function forms the basis for the unified and compact representation of other adaptive transmission schemes that we describe in Section $\mathrm{V}$

For a single user, the optimization problem of 5 can be rewritten as:

$$
\max _{r, p} \mu r-\lambda p
$$

Rewriting (12) in terms of the received power $q$, and channel gain $h$, we get

$$
\max _{r, q} \mu r-\lambda \frac{q}{h}
$$

subject to

$$
r \leq \frac{1}{2} \log _{2}\left(1+\frac{q}{\sigma^{2}}\right)
$$

Substituting (14) in (13) the optimization problem can be restated as

$$
\max _{q}\left[\mu \frac{1}{2} \log _{2}\left(1+\frac{q}{\sigma^{2}}\right)-\frac{\lambda}{h} q\right]
$$

Since

$$
\frac{1}{2} \log _{2}\left(1+\frac{q}{\sigma^{2}}\right)=\int_{0}^{q} \frac{1}{2\left(\sigma^{2}+z\right)} d z,
$$

we can rewrite (15) using (16) to get

$$
\max _{q} \int_{0}^{q}\left[\frac{\mu}{2\left(\sigma^{2}+z\right)}-\frac{\lambda}{h}\right] d z .
$$

In (17), $\left(\sigma^{2}+z\right)$ represents the interference level, the maximum value of which is equal to $\left(\sigma^{2}+q\right)$. The integrand in (17) is defined [3] to be the marginal utility function $u(z)$. Thus the optimization problem in terms of the marginal utility function can be written as

$$
\max _{q} \int_{0}^{q} u(z) d z
$$

It follows that the marginal utility function can be interpreted as the difference between the rate reward and the power cost.

\section{B. The marginal utility function in the energy harvesting case}

In the energy harvesting case, the power available at the transmitter may be lower than the power required by the optimal power adaptation. In such cases, the transmitter can only use the available energy from the battery for transmission. Representing the available energy in the battery as $b$ and accounting for the energy harvesting constraint, 17] can be rewritten as

$$
\max _{q^{e h}} \int_{0}^{q^{e h}}\left[\frac{\mu}{2\left(\sigma^{2}+z\right)}-\frac{\lambda}{h}\right] d z
$$

where

$$
q^{e h} \leq \frac{b h}{T} .
$$

Thus (18) can be written as

$$
\max _{q^{e h}} \int_{0}^{q^{e h}} u^{e h}(z) d z
$$

where $u^{e h}(z)$ is the marginal utility function with energy harvesting.

Then, in the multi-user case, for user $i$,

$$
u_{i}^{e h}(z)= \begin{cases}\frac{\mu_{i}}{2\left(\sigma^{2}+z\right)}-\frac{\lambda_{i}}{h_{i}}, & z \in A_{i} \\ 0, & z \notin A_{i}\end{cases}
$$

The rate and power allocation is then given by

$$
R_{i}(h)=\int_{A_{i}} \frac{1}{2\left(\sigma^{2}+z\right)} d z
$$

and

$$
P_{i}(h)=\left|A_{i}\right|
$$

where

$$
\begin{aligned}
A_{i} \equiv\left\{z \in[0, \infty): u_{i}^{e h}(z)\right. & >u_{j}^{e h}(z) \\
\forall j & \left.\neq i, u_{i}^{e h}(z)>0\right\}
\end{aligned}
$$

A four user example of how the marginal utility function for energy harvesting systems is selected to compute the power used and rate achieved is shown in Figure 1. The dashed lines in Figure 1 represent the marginal utility function $u^{e h}(z)$ of each user without taking interference into account. As can be seen from the Figure, User 3 has the highest non-zero marginal utility and so $u_{3}^{e h}(z)$ is first selected. The user with the next best $u^{e h}(z)$ is considered at an interference level where the previous user's $u^{e h}(z)$ becomes zero either due to the interference level or due to the lack of energy in it's battery. In this example, User 3's insufficient battery energy causes truncation of it's marginal utility. User 4 has the next best non-zero, marginal utility $u_{4}^{e h}(z)$, and so is shifted and 


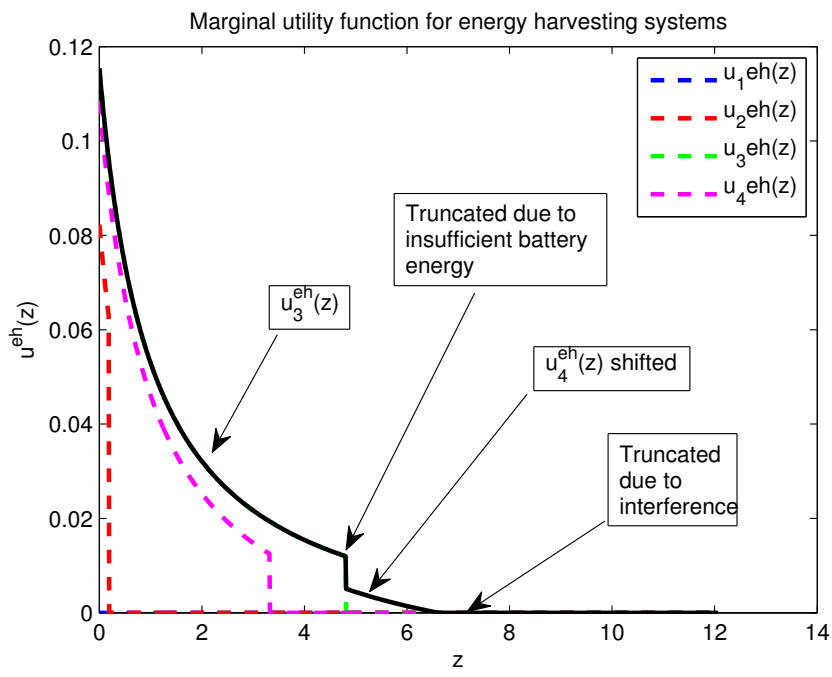

Fig. 1. Four user example. The marginal utility function of user $3, u_{3}^{e h}(z)$ is truncated due to lack of energy in its battery. The marginal utility function of user $4, u_{4}^{e h}(z)$ is truncated due to the interference level. The marginal utility function of users 1 is zero and that of user 2 falls below the interference level and are not selected.

selected at the point where $u_{3}^{e h}(z)$ is truncated. Next, User 4's marginal utility function becomes zero at the point shown in Figure 11. As far as the other two users are concerned, User 1 's marginal utility is zero and hence is not selected. User 2's marginal utility function falls below the interference level and is also not selected. This completes the selection of the $u_{i}^{e h}(z)$ for each user $i$. Then $A_{i}$ is obtained by picking at each interference level, the user with the highest marginal utility function. In this example, $A_{i}$ is region where the solid line is non-zero.

\section{Proportionally Fair Allocation And Index of FAIRNESS}

The optimal power allocation for the flat-fading MAC that achieves the maximum sum-rate point on the capacity region (often termed as the MaxSNR scheme) exhibits poor fairness towards the users. Users with poor channel conditions will not be serviced in a timely fashion. In practical systems with finite buffers, such users will experience more delay and possible buffer overflow. Additionally, in energy harvesting systems with finite batteries, harvested energy may be lost due to finite battery size if a user is not scheduled for transmission in an extended period of time.

\section{A. Proportionally fair rate allocation}

In order to address the lack of fairness associated with the MaxSNR scheme, the well known proportionally fair rate allocation scheme has successfully been used to improve fairness towards the users in the up-link. Rather than choosing the user with the 'absolute' best channel, the proportional fair scheduler selects the user with the best 'normalized' channel (i.e. the channel that is experiencing the best fading condition compared to its own average). Thus, this scheme maintains fairness towards the users, while also leveraging multiuser diversity.

The PF rate allocation scheme also offers the ability to trade-off rate with fairness which is not possible with the MaxSNR scheme. In Section V] we adapt the PF rate allocation scheme to energy harvesting systems and call it the PFEH scheme. We characterize the rate-fairness trade-off of this scheme. As will be seen in Section VI, this scheme is unable to achieve the range of rate-fairness trade-off of its non-energy harvesting equivalent under resource constrained settings. Hence we propose another scheme in Section V that improve rate-fairness trade-off achievable by the PFEH scheme, in various scenarios. Significantly, this improvement applies to non-energy harvesting systems as well.

\section{B. Jain Index of Fairness}

We next review the Jain Index of Fairness [9]. This index is used to quantify the fairness of the adaptive transmission schemes that we introduce in Section V

The Jain index of fairness, $I$, is defined as [9]:

$$
I=\frac{\left(\sum_{i}^{K} R_{i}\right)^{2}}{K * \sum_{i}^{K}\left(R_{i}\right)^{2}}
$$

where $R_{i}$ is the instantaneous rate of user $i$ and $K$ is the total number of users. If all $R_{i}$ are equal, then $I$ is equal to 1 . On the other hand, when the rate is allocated exclusively to one user among all the $K$ users, then $I$ is equal to $1 / K$, which is the least fair allocation.

This metric is well suited to study the fairness of resource allocation and has the following key advantages: (1) Population size independence: The index is applicable to any number of users. (2) Scale and metric independence: The index is independent of the quantity being measured. (3) Boundedness: The index is bounded between 0 and 1. (4) Continuity: The index is continuous.

\section{LOW-DELAY ADAPTIVE TRANSMISSION STRATEGIES}

In this section, we consider several low delay adaptive transmission strategies to illustrate the importance of jointly using the knowledge of the channel state, battery conditions, instantaneous fairness values and allowing more than one user to access the channel, in energy harvesting resource constrained settings. To show why joint consideration of these system parameters is important, we also describe schemes that pick only one user to transmit, based on either the best channel or the best battery-channel product for comparison with schemes that allow groups of users to compete for channel access. However, all these schemes exhibit poor fairness towards the users. So we adapt the PF scheme to energy harvesting systems. We finally propose another scheme that offers better rate-fairness trade-off than PFEH under various scenarios considered in Section VI

All of the low delay adaptive transmission strategies in this Section are described using the framework and notation developed in terms of the energy harvesting equivalent of the marginal utility function developed in Section III (see (22a), 22b)). In each of the schemes, the rate and power allocation are given respectively by (23) and (24). 


\section{A. Single-user channel adaptive transmission - (SUCA)}

In this adaptive transmission strategy, the receiver selects the user $i$ with the best channel, i.e. $i=\arg \left(\max _{j}\left[h_{j}\right]\right)$. For this user $i$,

$$
A_{i}^{\text {suca }} \equiv\left\{z \in[0, \infty): u_{i}^{e h}(z)>0\right\}
$$

and

$$
A_{k}^{\text {suca }} \equiv\{\emptyset\}, \forall k \neq i
$$

This single-user channel adaptive transmission, which considers the channel state alone, results in lower rate, when the nodes are equipped with small size batteries. Hence we consider other schemes such as single-user channel-battery product (SUCA) scheme and a scheme where a group of users transmit simultaneously (GPCA).

\section{B. Single-user (battery-channel) product adaptive transmis- sion - (SUPA)}

In this adaptive transmission strategy, the receiver selects the user $i$ with the best battery-channel product, i.e. $i=$ $\arg \left(\max _{j}\left[b_{j} h_{j}\right]\right)$. As a result, the user with the best channel may not always get selected. For user $i$,

$$
A_{i}^{\text {supa }} \equiv\left\{z \in[0, \infty): u_{i}^{e h}(z)>0\right\}
$$

and

$$
A_{k}^{\text {supa }} \equiv\{\emptyset\}, \forall k \neq i
$$

\section{Group-power channel adaptive transmission - (GPCA)}

In this adaptive transmission strategy, the receiver first selects the user with the highest marginal utility function $u^{e h}(z)$. Starting with this user, additional users are selected such that there is a net positive increase in the overall objective function given by (5). Starting at $z=0$, at each interference level $\sigma^{2}+z$, users are added to get the largest positive marginal increase in the objective function given by $\mu \mathbf{r}-\lambda \mathbf{p}$. Since $u^{e h}(z)$ represents this objective function, selecting users with the largest positive marginal increase in the objective function is equivalent to picking the users with the highest $u^{e h}(z)$ at each interference level. Transmission is then done by the group of users selected. Specifically,

$$
\begin{aligned}
& A_{i}^{\text {gpca }} \equiv\{z \in[0, \infty): u_{i}^{e h}(z)>u_{j}^{e h}(z) \\
&\left.\forall j \neq i, u_{i}^{e h}(z)>0\right\}
\end{aligned}
$$

\section{Proportionally fair energy harvesting adaptive transmis-} sion - (PFEH)

In this adaptive transmission strategy, the user with the highest normalized rate is scheduled for transmission. The average throughput of each user in a past window of size $t_{w}$ is computed. The window size can be used to trade-off rate and fairness, with smaller $t_{w}$ ensuring better fairness towards the users, as shown in Section VI. For the most fair allocation, the window size is set equal to the number of users $K$.

Denoting the selected user at the $n^{\text {th }}$ time instant as $i$, the highest normalized rate is given by

$$
\xi_{i}=\frac{R_{i}(n)}{W_{i}(n)}
$$

where $R_{i}$ is the current rate achievable using (23) by setting the $u_{j}^{e h}(z)=0, \forall j \neq i$ and $W_{i}$ is the $i^{\text {th }}$ user's average throughput in the previous window. The update of the average throughput for the next time instant is given by

$$
W_{j}(n+1)=\left\{\begin{aligned}
\left(1-\frac{1}{t_{w}}\right) W_{j}(n) & j=i \\
+\frac{1}{t_{w}} R_{j}(n) & \\
\left(1-\frac{1}{t_{w}}\right) W_{j}(n), & j \neq i .
\end{aligned}\right.
$$

Thus, the receiver selects user $i$ with the highest normalized rate, i.e. $i=\arg \left(\max _{j}\left[\xi_{j}\right]\right)$. For this user $i$,

$$
A_{i}^{p f e h} \equiv\left\{z \in[0, \infty): u_{i}^{e h}(z)>0\right\}
$$

and

$$
A_{k}^{p f e h} \equiv\{\emptyset\}, \forall k \neq i
$$

\section{E. Weighted proportionally fair energy harvesting adaptive transmission - (WPFEH)}

Although the proportionally fair scheme PFEH, described in the previous section, allows trade-off of rate and fairness, the range of trade-off is diminished in resource constrained settings. Allowing all users to compete for channel access improves the range of rate-fairness trade-off. Further, preferentially weighting the users improves the range of this trade-off even more, motivating the WPFEH scheme. This scheme is similar to the GPCA scheme, in that all users are allowed to compete for channel access, except that they are preferentially weighted according to any desired metric. For instance a weighting based on the proportionally fair metric is given by

$$
\mu_{i}= \begin{cases}\frac{(1+p)}{K}, & i=\max _{i}\left[\xi_{i}\right] \\ 1-\frac{1+p}{K}, & j \neq i .\end{cases}
$$

where $0<p<1$ can be chosen appropriately to achieve different points on the rate-fairness boundary as discussed in Section VI This scheme can be represented by

$$
\begin{aligned}
A_{i}^{\text {wpfeh }} \equiv\{z \in[0, \infty) & : u_{i}^{e h}(z)>u_{j}^{e h}(z) \\
& \left.\forall j \neq i, u_{i}^{e h}(z)>0\right\}
\end{aligned}
$$

\section{Numerical Results}

In this section, we evaluate the performance of the various schemes under different scenarios via simulation. The simulation is run for small and large battery sizes as well as for varying recharge rates (or equivalently, SNR) of the harvesting process. For the simulation, we sample 900,000 points of a Rayleigh distribution for the channel and the same number of samples of a first order Markov process for the energy harvesting process. Stochastic models are typically used to describe the unpredictable nature of energy harvesters. In [23] solar energy is modeled as a Markov process after analysis of years of observed data. In this work, we use a 10-state model with transition probabilty matrix as given in [24] and a similar energy profile. With solar energy, changes in the 
amount of energy harvested occurs at a time scale much slower than the changes in the channel gain. However, for other types of energy harvesting sources, such as harvesters based on vibration energy, the changes in the amount of energy harvested may occur in the same time scale as the changes in the channel gain. Numerical results for the latter scenario can be found in [25].

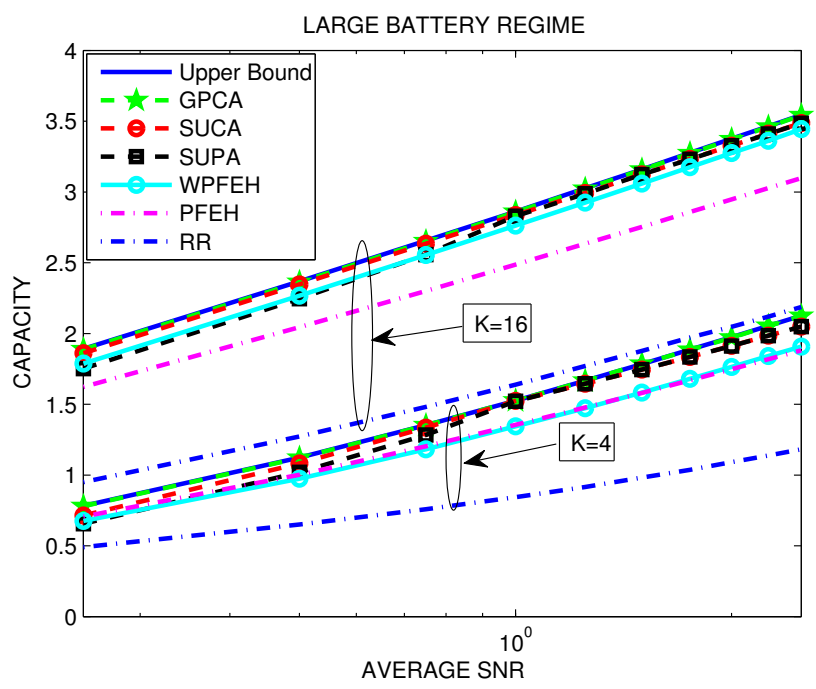

Fig. 2. $C$ versus $S N R$ for $K=4,16$. Each user's battery size is fixed at $1 e^{6} \mathbf{J}$.

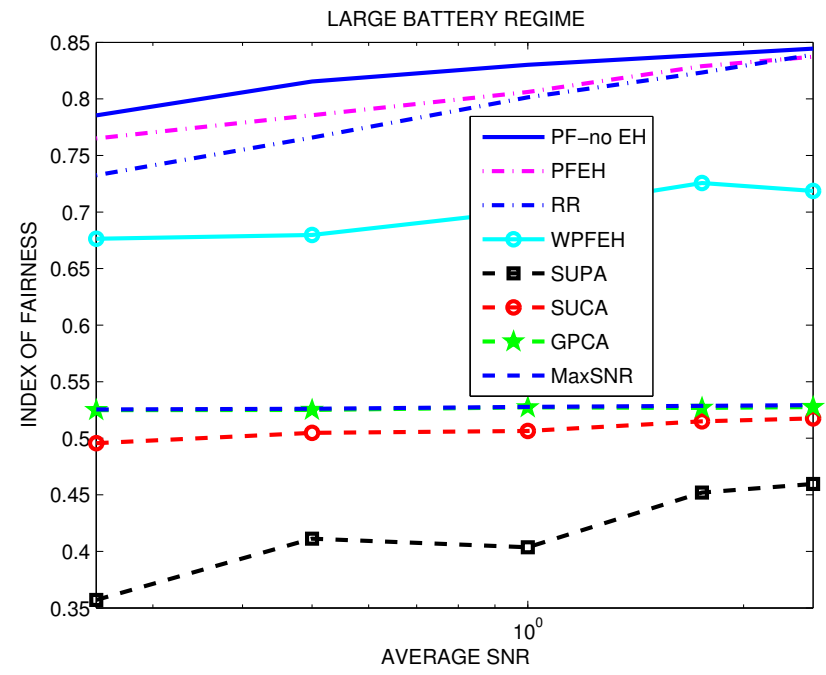

Fig. 3. $I$ versus $S N R$ for $K=16$. The battery size of each user is fixed at $1 e^{6} \mathbf{J}$.

Figure 2 shows the rate achieved by the different schemes with varying recharge rate in the large battery regime for $\mathrm{K}=4$ and 16 users. The GPCA scheme achieves rates very close to the upper bound while the round robin scheme (RR) achieves the lowest rate compared to all other schemes. The single user schemes SUPA and SUCA achieve rates close to GPCA at higher recharge rates. Schemes based on the proportionally fair metric (PFEH and WPFEH) achieve rates between GPCA and RR.
Figure 3 shows the fairness achieved by the different schemes with varying recharge rate in the large battery regime for $\mathrm{K}=16$ users. The multi-user, channel-based GPCA scheme achieves fairness very close to the non-energy harvesting MaxSNR scheme. At lower recharge rates, the energy level in the battery has a greater influence on the user selection. Hence the battery-channel product based SUPA scheme achieves worse fairness than the SUCA scheme, which is the energy harvesting equivalent of the MaxSNR scheme. As the recharge rate increases, the channel gain has a greater influence on the user selection and hence the fairness of the SUPA scheme approaches the channel based schemes. The PFEH scheme maintains fairness close to the non-energy harvesting equivalent scheme (shown as solid line in the Figure) for the range of recharge rates considered. The scheme WPFEH achieves much better fairness towards the users although the rate achieved is close to GPCA, SUPA and SUCA as can be seen from Figure 2 . The round robin scheme achieves better fairness than the WPFEH scheme, but the rate achieved is lower than all the other schemes.

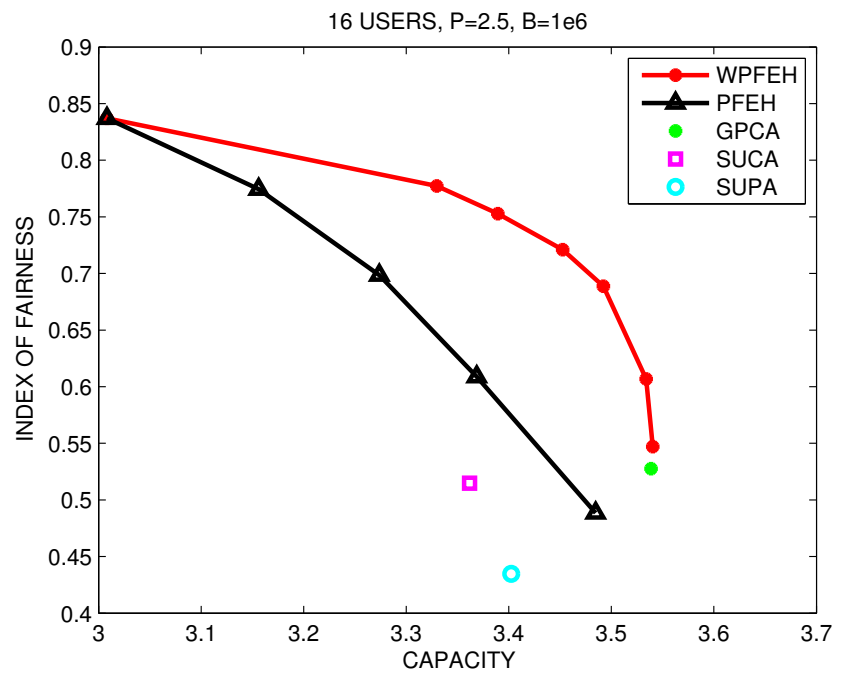

Fig. 4. $I$ versus $C$ for $K=16$ and SNR $=2.5$ Watts. The battery size of each user is fixed at $1 e^{6}$ Joules.

Figure 4 shows the trade-off between rate and fairness for the various schemes in the large battery regime for $\mathrm{K}=16$ users with an average recharge rate of 2.5 Watts for all users. Single user schemes SUPA and SUCA, as well as the group scheme GPCA can achieve only a single point on the rate-fairness region. Schemes based on the proportionally fair allocation achieve good trade-off on the rate-fairness region. The WPFEH scheme achieves better rate-fairness trade-off than PFEH. These results hold even for non-energy harvesting systems and are not shown due to similarity in the nature of the rate-fairness trade-off. The round robin scheme is not shown here since the rate-fairness point achieved is far inferior to any of the other schemes.

Figure 5 shows the rate achieved by the different schemes with varying recharge rate in the small battery regime for $\mathrm{K}=4$ and 16 users. At the lowest SNR, the rate achieved by all the schemes is similar to that achieved in the large battery 


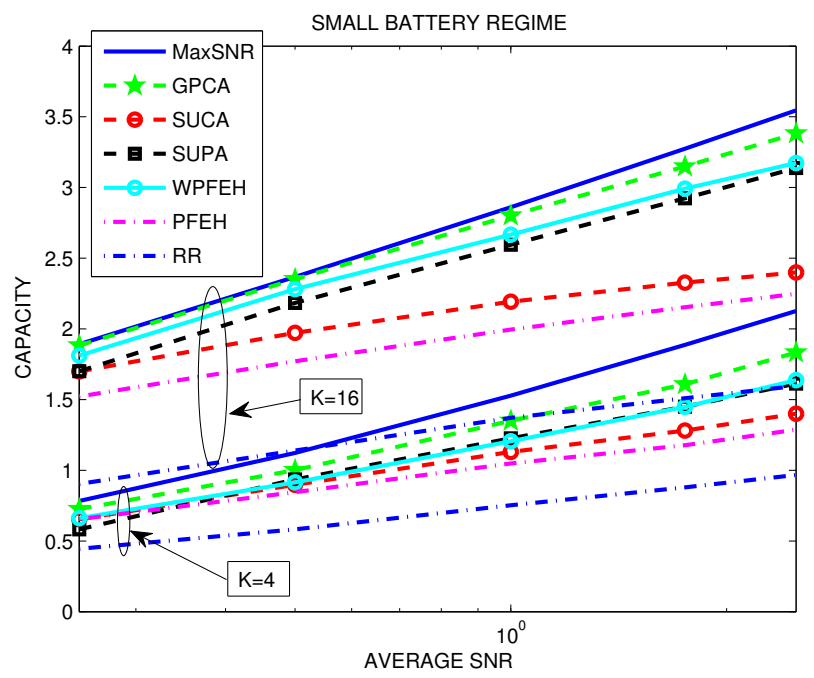

Fig. 5. $C$ versus $S N R$ for $K=4,16$. The battery size of each user is fixed at $1 e^{3}$ Joules.

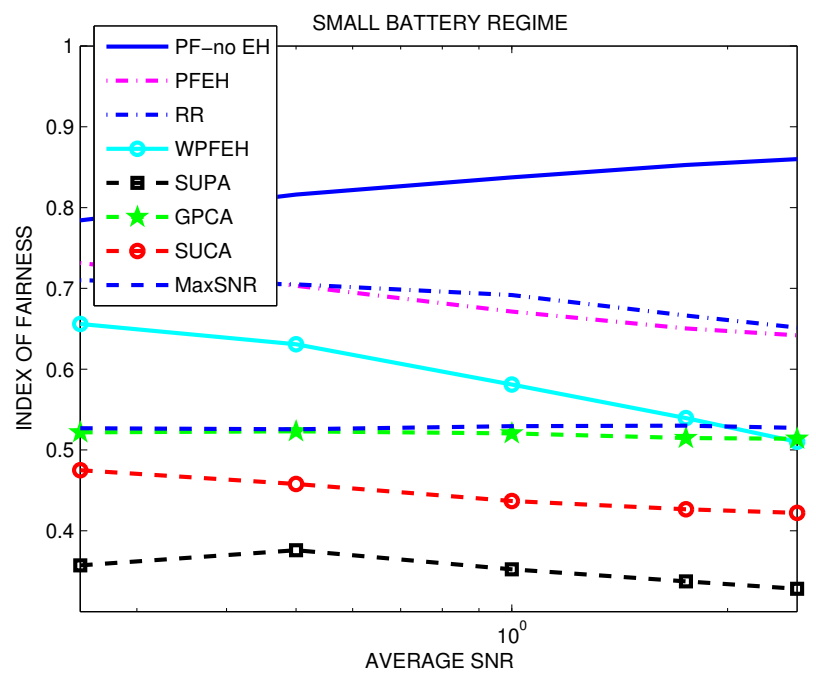

Fig. 6. $I$ versus $S N R$ for $K=16$. The battery size of each user is fixed at $1 e^{3}$ Joules.

regime as in Figure 2. The drop in performance is however more pronounced for higher recharge rates. The GPCA scheme outperforms all other schemes in this scenario. However SUPA and WPFEH achieve rates close to GPCA. In general, schemes that select a group of users (GPCA, WPFEH) and schemes that take the battery state in to account (SUPA), achieve higher rate in the small battery regime.

Figure 6 shows the fairness achieved by the different schemes with varying recharge rate in the small battery regime for $\mathrm{K}=16$ users. The fairness of all the schemes decreases with increase in recharge rate. The GPCA scheme shows the least variation in fairness with increasing recharge rate. The WPFEH scheme shows large variation in fairness and falls below the GPCA scheme at larger recharge rates. Although the round robin scheme achieves better fairness than PFEH, the rate achieved is lower than all the other schemes as shown in the Figure 5.

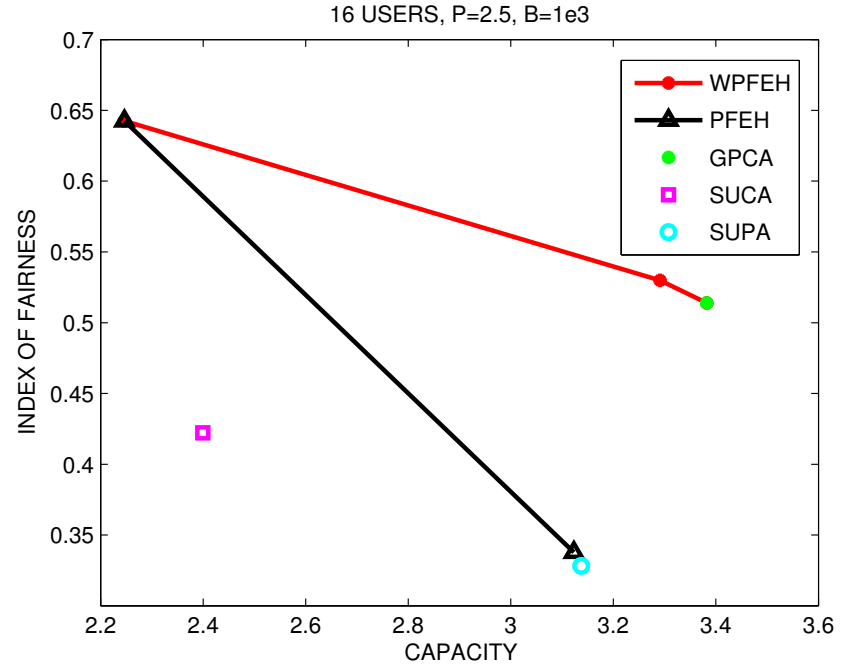

Fig. 7. $I$ versus $C$ for $K=16$ and SNR $=2.5$ Watts. The battery size of each user is fixed at $1 e^{3}$ Joules.

Figure 7 shows the trade-off between rate and fairness for the various schemes in the small battery regime for $\mathrm{K}=16$ users for a fixed average recharge rate of 2.5 Watts for all users. Among the schemes developed in the spirit of the MaxSNR scheme, the group scheme GPCA achieves a ratefariness point that is better than that achieved either by SUPA or SUCA. Similar to the large battery regime, schemes based on the proportionally fair allocation (PFEH, WPFEH) achieve various points on the rate-fairness region. The WPFEH scheme performs better than the PFEH scheme in the small battery regime as well, because all users are allowed to compete for channel access at all times.

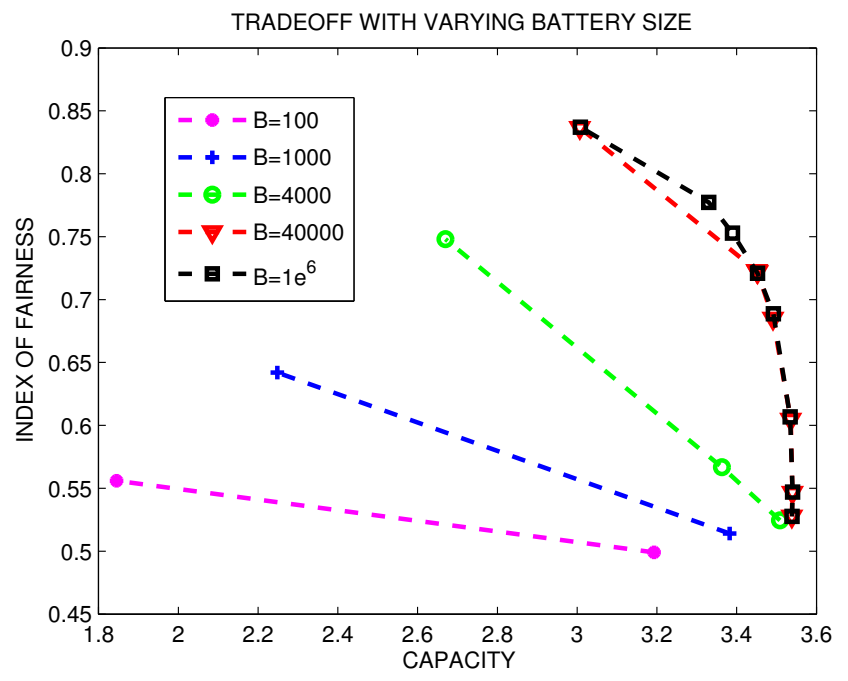

Fig. 8. Trade-off achieved between rate and fairness for various battery sizes. The symmetric case with $\mathrm{K}=16$ users with recharge rate of $2.5 \mathrm{~W}$ is shown.

Figures 8 shows the rate-fairness trade-off that can be achieved with varying battery size under different recharge rates. From Figure 8 it can be seen that a battery sizes greater than or equal to 40000 Joules is required to achieve a good 
range of rate-fairness trade-off in the $\mathrm{K}=16$ user symmetric case.

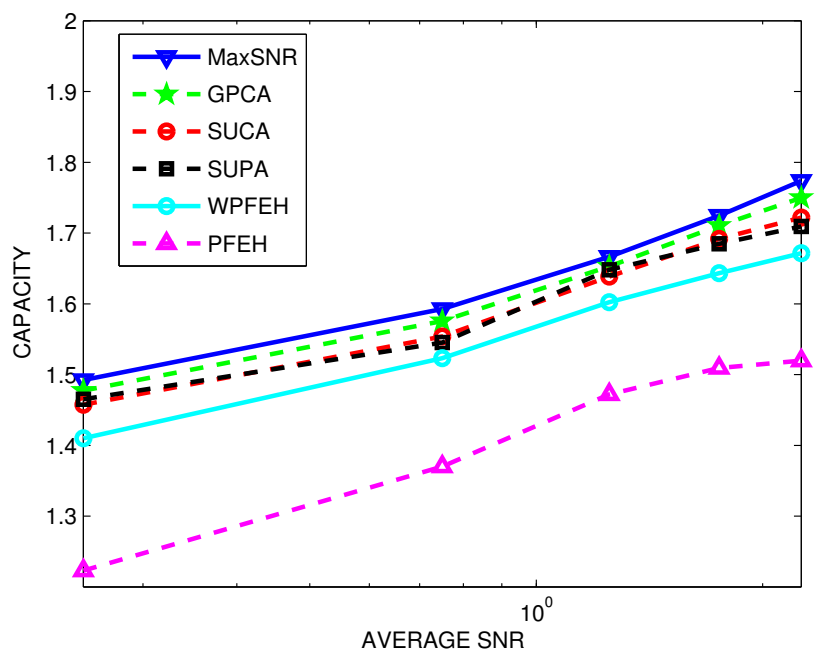

Fig. 9. $C$ versus $S N R$ for $K=4$. Performance of adaptive transmission schemes for the asymmetric case. The SNR (average recharge rate) of three users fixed at 1.25 Watts. The recharge rate of the fourth user is varied from .25 Watts to 2.25 Watts. The battery size of each user is fixed at $1 e^{6}$ Joules.

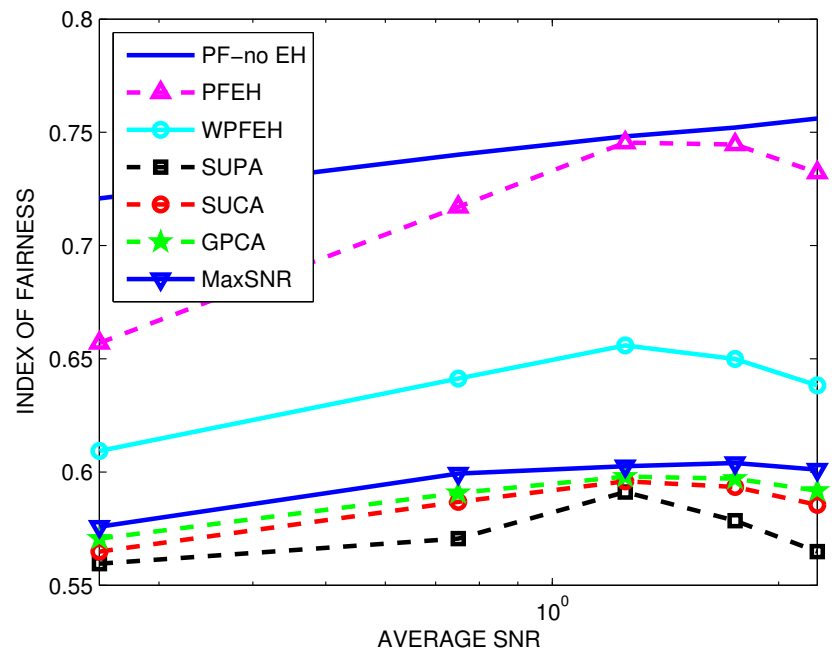

Fig. 10. $I$ versus $S N R$ for $K=4$. Performance of adaptive transmission schemes for the asymmetric case. The SNR (average recharge rate) of three users fixed at 1.25 Watts. The recharge rate of the fourth user is varied from .25 Watts to 2.25 Watts. The battery size of each user is fixed at $1 e^{6}$ Joules.

So far we have characterized the performance of symmetric users. We now study the performance of asymmetric users in the large battery regime. We let three users have the same average recharge rate of $1.25 \mathrm{~W}$ and call them identical users. In Figures 9 and 10, we allow the recharge rate of the 4th user to vary from $0.25 \mathrm{~W}$ to $2.25 \mathrm{~W}$. Shown in Figure 9 is the sum-rate of all four users. Figure 10 shows the variation of the index of fairness with varying recharge rate of user 4 . As in the symmetric case, the schemes based on the proportionally fair method offer better fairness at the expense of lower rate. The fairness of all the schemes reach their maximum point when the recharge rate of user 4 is equal to that of the other users, i.e. at $1.25 \mathrm{~W}$, as can be seen from Figure 10.

In summary, we highlight the features of some of the schemes. The scheme GPCA achieves near optimal performance in terms of achieving the sum-rate point, in the large battery regime, thereby showing that the MaxSNR equivalent scheme for low delay energy harvesting networks, is not a single user scheme, but rather a group scheme that can obtain energy from a group of users. The scheme SUPA and GPCA achieve higher rate than SUCA in the small battery regime, thereby showing the importance of considering the battery state in addition to the channel state in resource constrained settings. The scheme WPFEH offers better fairness than all the schemes developed in the spirit of the MaxSNR scheme, at a marginal reduction in rate. Further, the scheme WPFEH achieve points above the convex hull of the points achieved by PFEH in the large battery regime.

\section{CONCLUSIONS}

In this paper we obtained the AWGN-MAC capacity region with energy harvesting transmitters. We studied three low delay, adaptive transmission schemes that acheived capacity close to the maximum sum-of-rates capacity under resource constrained settings. To overcome the unfair rate allocation inherent to the sum-of-rates allocation, we adapted the proportionally fair (PF) rate allocation to energy harvesting nodes. We developed new adaptive transmission strategies based on the PF rate allocation that achieved points above the ratefairness boundary of the convex hull of the PF rate allocation. We characterized performance of all the adaptive transmission schemes through extensive simulation.

In this work we studied rate-fairness trade-offs in a Multiple Access Channel where each transmitter is equipped with an energy harvester and all the harvested energy is assumed to be available for transmission. However in low power energy harvesting systems, significant energy costs may be incurred to power circuitry and for data processing. In addition, battries may have storage inefficiences. These effects could be included in future analysis.

\section{REFERENCES}

[1] C. Shannon, "A mathematical theory of communication," The Bell System Technical Journal, vol. 27, no. 7, pp. 379-423, 1948.

[2] E. Biglieri, J. Proakis, and S. Shamai, "Fading channels: Informationtheoretic and communications aspects," IEEE Transactions on Information Theory, vol. 44, no. 6, pp. 2619-2692, 1998.

[3] D. Tse and S. Hanly, "Multiaccess fading channels. I. polymatroid structure, optimal resource allocation and throughput capacities," IEEE Transactions on Information Theory, vol. 44, no. 7, pp. 2796-2815, 1998.

[4] R. Knopp and P. Humblet, "Information capacity and power control in single-cell multiuser communications," in IEEE International Conference on Communications, 1995. ICC'95 Seattle,'Gateway to Globalization', 1995, vol. 1. IEEE, 1995, pp. 331-335.

[5] P. Viswanath, D. Tse, and R. Laroia, "Opportunistic beamforming using dumb antennas," IEEE Transactions on Information Theory, vol. 48 , no. 6, pp. 1277-1294, 2002.

[6] O. Ozel and S. Ulukus, "Information-theoretic analysis of an energy harvesting communication system," in 2010 IEEE 21st International Symposium on Personal, Indoor and Mobile Radio Communications Workshops (PIMRC Workshops). IEEE, 2010, pp. 330-335.

[7] - "Achieving AWGN capacity under stochastic energy harvesting," Preprint, 2011. 
[8] R. Rajesh, V. Sharma, and P. Viswanath, "Information capacity of energy harvesting sensor nodes," Arxiv preprint arXiv:1009.5158, 2010.

[9] R. Jain, D. Chiu, and W. Hawe, "A quantitative measure of fairness and discrimination for resource allocation in shared computer systems," DEC Research Report TR-301, 1984.

[10] D. Bartolomé, T. Sartenaer, J. Gómez, L. Vandendorpe, and A. PérezNeira, "Fairness at the physical layer: Overview, tools, and applications."

[11] A. Goldsmith and P. Varaiya, "Capacity of fading channels with channel side information," IEEE Transactions on Information Theory, vol. 43, no. 6, pp. 1986-1992, 1997.

[12] A. Goldsmith, Wireless communications. Cambridge Univ Press, 2005.

[13] V. Sharma, U. Mukherji, and V. Joseph, "Efficient energy management policies for networks with energy harvesting sensor nodes," in 46th Annual Allerton Conference on Communication, Control, and Computing. IEEE, 2008, pp. 375-383.

[14] K. Kumaran and L. Qian, "Uplink scheduling in CDMA packet-data systems," in INFOCOM 2003. Twenty-Second Annual Joint Conference of the IEEE Computer and Communications. IEEE Societies, vol. 1. IEEE, 2003, pp. 292-300.

[15] L. Yang, M. Kang, and M. Alouini, "On the capacity-fairness tradeoff in multiuser diversity systems," IEEE Transactions on Vehicular Technology, vol. 56, no. 4, pp. 1901-1907, 2007.

[16] R. Rajesh and V. Sharma, "Capacity of fading Gaussian channel with an energy harvesting sensor node," Arxiv preprint arXiv:1010.5416, 2010.

[17] J. Yang and S. Ulukus, "Optimal packet scheduling in a multiple access channel with rechargeable nodes," in Communications (ICC), 2011 IEEE International Conference on. IEEE, 2011, pp. 1-5.

[18] C. Huang, R. Zhang, and S. Cui, "Throughput maximization for the Gaussian relay channel with energy harvesting constraints," Arxiv preprint arXiv:1109.0724, 2011.

[19] O. Ozel, J. Yang, and S. Ulukus, "Optimal broadcast scheduling for an energy harvesting rechargeable transmitter with a finite capacity battery," IEEE Trans. Wireless Comm, 2011.

[20] K. Tutuncuoglu and A. Yener, "Sum-rate optimal power policies for energy harvesting transmitters in an interference channel," Arxiv preprint arXiv:1110.6161, 2011.

[21] O. Ozel, K. Tutuncuoglu, J. Yang, S. Ulukus, and A. Yener, "Transmission with energy harvesting nodes in fading wireless channels: Optimal policies," IEEE Journal on Selected Areas in Communications, vol. 29, no. 8, 2011.

[22] M. Shaqfeh and N. Goertz, "Comments on the boundary of the capacity region of multiaccess fading channels," IEEE Transactions on Information Theory, vol. 55, no. 7, pp. 3407-3408, 2009.

[23] P. Poggi, G. Notton, M. Muselli, and A. Louche, "Stochastic study of hourly total solar radiation in corsica using a Markov model," International Journal of Climatology, vol. 20, no. 4, pp. 1843-1860, November 2000.

[24] A. E. Susu, A. Acquaviva, D. Atienza, and G. De Micheli, "Stochastic modeling and analysis for environmentally powered wireless sensor nodes." WiOpt, 2008.

[25] R. Raghuvir, "Adaptive transmission strategies in energy harvesting wireless networks," Ph.D. dissertation, Southern Methodist University, 2012. 